\title{
Could targeted, antibiotic-loaded gold nanoconstructs be a new magic bullet to fight infection?
}

\author{
“...antibiotic-loaded, targeted AuNCs are capable \\ of eliminating viable bacteria from both planktonic \\ cultures as well as within biofilms..."
}

First draft submitted: 1 July 2016; Accepted for publication: 20 July 2016; Published online: 16 August 2016

The concept of a 'magic bullet' for use in the fight against human diseases as introduced by Paul Ehrlich has inspired generations of scientists to invent potent molecular therapeutics targeting pathogens without harming healthy tissues [1]. Many synthetic compounds have been discovered with cell-structural targets based on the understanding of the interface between chemistry, biology and medicine. Nanomedicine offers the advantage of nanocarriers and nanotechnology to deliver these compounds to sites of pathology with minimum side effects. Gold nanoparticles have been extensively explored in this regard for more than a decade owing to their bioinert nature, facile surface modification and unique optical properties.

In fact, long before the 'nanotechnology' era, elemental gold and gold compounds were utilized by medical practitioners for the treatment of various pathologies. For instance, gold treatment has been used successfully in the management of rheumatoid arthritis since its introduction by Jacques Forestier over 80 years ago [2], although it is not well understood how gold salts work in this context beyond the fact that they reduce inflammation in the joint. Radioactive colloidal gold $\left({ }^{198} \mathrm{Au}\right)$ therapy was introduced by Rubin Flocks over 60 years ago to treat prostate cancer. His technique evolved over nearly 25 years with the treatment of 1515 patients, and short- and long-term outcomes suggest that the use of colloidal gold resulted in statistically significant improvements in survival compared with other contemporary theory that colloidal gold provides localized radiation with less risk to adjacent structures and transportation to draining lymph nodes for the treatment of metastasized tumor cells.

Since that time, technology has advanced to the point of using gold nanoparticles as carriers to deliver therapeutic agents. Indeed, one such formulation is undergoing clinical trials in humans. This formulation contains recombinant human TNF- $\alpha$ and thiolyated polyethylene glycol conjugated to the surface of $27-\mathrm{nm}$ colloidal gold nanoparticles. The results of Phase I clinical studies showed targeted accumulation within tumors compared with native recombinant human TNF- $\alpha$ following intravenous administration [4]. This formulation has now been combined with approved chemotherapies in Phase II clinical trials for the systemic treatment of nonresectable cancers.

An additional advance is the ability to manipulate gold nanoparticles using exposure to light. The manipulation of metal nanoparticles with light dates to the 4th century AD during the Roman period with the fabrication of the Lycurgus Cup. In nanomedicine, the light absorption by gold nanoparticles has been utilized to treat diseases. Specifically, laser-irradiated gold nanoparticles can generate sufficient heat to achieve a lethal photothermal (PT) effect, leading to the treatments [3]. These results support Flocks'

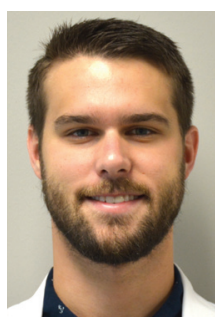

Daniel G Meeker

Department of Microbiology \& Immunology, University of Arkansas for Medical Sciences, Little Rock, AR 72205 , USA

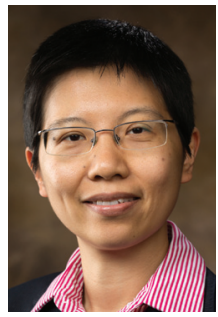

Jingyi Chen Author for correspondence: Department of Chemistry and Biochemistry, University of Arkansas, Fayetteville, AR 72701, USA chenj@uark.edu

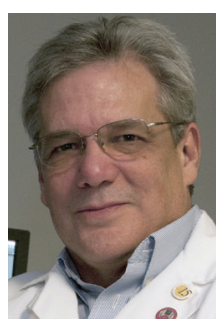

Mark S Smeltzer

Author for correspondence: Department of Microbiology \& Immunology, University of Arkansas for Medical Sciences, Little Rock, AR 72205, USA

SmeltzerMarkS@uams.edu

Future $\because \%$ Medicine part of 
physical destruction of target cells [5]. One such technology (AuroLase) based on gold nanoshells and nearinfrared (NIR) laser irradiation pioneered by Halas [6] is currently undergoing pilot clinical studies for the treatment of head and neck and lung cancer patients.

To date, the primary clinical application of nanotechnology has focused on the treatment of cancer [7]. In contrast, nanotechnology applications in the context of infectious disease have been extremely limited, with Shi and colleagues reporting in 2014 that only 14 of approximately 100 nanomedicine products marketed for bacterial diagnosis, antibiotic delivery and the development of antimicrobial medical devices [8]. Despite this disparity, the field of infectious disease stands to benefit greatly from advances in nanomedicine for a number of reasons. The first and arguably most important of these is that, since the advent of the 'antibiotic era', the emergence of antibiotic resistance has quickly out-paced the development of novel traditional antimicrobial drugs, a fact that is exacerbated by the decreased effort by the pharmaceutical industry to develop new antibiotics [9]. This problem is further exacerbated by the fact that many pathogenic bacteria are capable of forming a biofilm, an 'alternative lifestyle' that confers a therapeutically relevant level of intrinsic resistance to all currently available antibiotics $[10,11]$.

\section{"...the field of infectious disease stands to benefit greatly from advances in nanomedicine...}

Thus, biofilm-associated infections respond poorly to conventional antibiotic therapy irrespective of the acquired resistance status of the offending bacterial strains. This dictates the need for therapeutic measures that extend far beyond systemic antibiotic treatment [12]. In the case of orthopedic infections, this is reflected in the need for surgical intervention to debride and remove infected tissues and/or implants [13]. This intrinsic resistance suggests that the development of new antibiotics is unlikely to adequately address the clinical problem of biofilm-associated infections. The clinical significance of this is further emphasized by the fact that it has been estimated $80 \%$ of human bacterial infections are associated with the formation of a biofilm [14]. Thus, there is an urgent need for the development of alternative therapeutic approaches that can be used to combat both acquired and intrinsic antibiotic resistance. It is for these reasons that the nanomedicine field has begun to explore solutions to the problem of acquired antibiotic resistance and the intrinsic resistance of biofilm-associated infections [15].

The advantage of nanomedicine in the clinical context of infectious disease is that it offers the opportu- nity for the physical destruction of bacterial cells irrespective of their metabolic or even antibiotic resistance status. Among the first to explore the application of PT therapy in the context of infectious disease were Smeltzer and Zharov [16,17]. In these studies, Staphylococcus aureus was used as a proof-of-principle pathogen to establish the utility of PT therapy in the context of bacterial infection. To achieve a targeted approach, gold nanoparticles were conjugated to antibodies specific for the $S$. aureus surface proteins staphylococcal protein A (Spa) and a surface-associated lipoprotein of unknown function. It was demonstrated that pulsed NIR irradiation of gold nanoparticles attached to the $S$. aureus cell surface leads to the formation of 'nanobubbles,' disruption of the cell wall/membrane and ultimately bacterial cell death. Not only was this PT-mediated killing demonstrated in vitro [16], it was demonstrated that this approach was sufficient to reduce the number of viable bacteria in a murine bacteremia model below the limit of detection following transdermal laser irradiation [17]. These results demonstrate the therapeutic potential in the clinical context of bacteremia, but they do not address the potential of this approach in the context of biofilm-associated infections. In fact, in preliminary studies in which we assessed this approach using a catheter-associated model of biofilm infection, we did observe a significant PT effect, but it was not sufficient to completely eradicate viable bacteria from the biofilm.

As noted above, one of the ways in which nanotechnology is changing the field of medicine is the use of nanoparticles as drug-delivery vehicles, the goal being to achieve the controlled release of bioactive agents directly at the intended target. Many controlled release systems have been developed in the past two decades including the use of liposomes or polymers as nanocarriers for drug molecules. Since 1995, several of the liposomal and polymeric formulations have been approved and used in a clinical setting to treat cancer [18]. More recently, the design of drug delivery systems has been further advanced by incorporating 'smart' polymers from which the release of drug molecules is triggered by internal stimuli (e.g., temperature rise, $\mathrm{pH}$ change or small molecule or enzyme presence) or external stimuli (e.g., heat, light, electric or magnetic field) [19]. Thus, integration of 'smart' polymers with gold nanoparticles as a gold nanoconstruct platform could enable the controlled release of encapsulated antibiotics triggered by NIR laser irradiation owing to the PT effects on the gold nanostructures.

Therefore, we sought to exploit the properties of gold nanoparticles to create targeted, drug-delivery vehicles that could simultaneously generate PT effects and antibiotic release directly at the bacterial cell sur- 
face in a controlled fashion [20]. To achieve this goal, we constructed gold nanocages (AuNCs), which were then coated in a polydopamine (PDA) polymer coating. PDA coating allowed for both the incorporation of the membrane-active antibiotic, daptomycin and the conjugation of antibodies specific for Spa. Imaging studies confirmed that anti-Spa antibodies allowed for the targeting of AuNCs directly to bacterial cells. It was demonstrated that daptomycin was released from AuNCs in a controlled, laser-dependent manner following conformational changes in the PDA coating generated by the PT effect. In studies with the proofof-principle pathogen, $S$. aureus, we demonstrated that antibiotic-loaded, targeted AuNCs are capable of eliminating viable bacteria from both planktonic cultures as well as within biofilms in vitro when irradiated with a continuous wave NIR laser. Importantly, the PT-mediated killing effects and the bactericidal effects of released daptomycin demonstrated synergism. Specifically, in the context of a biofilm, the reduction in bacterial viability due to thermally-released antibiotic was significantly greater than the reduction due to free antibiotic alone at the same concentration. This is presumably due to PT-mediated physical destruction but could also be related to the impact of heat on the biofilm, which possibly disrupts the biofilm to allow greater penetration of antibiotic. The multimodality of our approach and the synergism of these modalities may also allow for the eradication of bacteria while minimizing the doses of each modality. In other words, lower doses of antibiotic and laser irradiation may be sufficient to eradicate infection when employed together, thus potentially minimizing the collateral damage and risk to the patient.

$$
66
$$

\section{demonstrated with both a methicillin-sensitive}

\section{S. aureus strain and a methicillin-resistant} S. aureus strain as well as within the context of
an established biofilm."

We believe that our results demonstrate the feasibility of our approach using the targeted, antibiotic-loaded, irradiated nanoconstruct. These findings warrant the further examination of its efficacy in vivo in both the context of bacteremia and biofilm-

\section{References}

1 Strebhardt K, Ullrich A. Paul Ehrlich's magic bullet concept: 100 years of progress. Nat. Rev. Cancer 8, 473-480 (2008).

2 Forestier JM. Bull. Mém. Soc. Méd. Hôp. Paris 53, 323 (1929).

3 Rosevear HM, Lightfoot AJ, O’Donnell MA,Platz CE, Loening SA, Hawtrey CE. Flocks and colloidal gold treatments for prostate cancer. Sci. World J. 11, 1560-1567 (2011). associated infection. The ultimate goal of our work is to develop a broadly applicable nanotherapeutic approach to infectious disease that demonstrates utility even against pathogens that exhibit acquired antibiotic resistance or intrinsic resistance associated with the formation of a biofilm. Indeed, the efficacy of our nanodrug was demonstrated with both a methicillinsensitive $S$. aureus strain and a methicillin-resistant $S$. aureus strain as well as within the context of an established biofilm. To date, the efficacy of our design has been demonstrated in $S$. aureus alone. However, an appealing aspect of our design is that the approach has the potential to be expanded to account for alternative bacterial pathogens. In principle, alternative bacterial pathogens could be targeted by substituting an effective antibiotic and an appropriate targeting agent (antibody, peptide, etc.). Thus, we believe the concept of using photoactivatable nanodrugs has tremendous potential to overcome the growing problem of acquired antibiotic resistance in bacteria as well as the intrinsic resistance of biofilm-associated infections.

\section{Disclaimer}

The content is solely the responsibility of the authors and does not necessarily represent the views of the $\mathrm{NIH}$.

\section{Financial \& competing interests disclosure}

This work was supported by the pilot project fund from the Arkansas Bioscience Institute and startup funds from the University of Arkansas to J Chen, as well as grant R56-Al093126 from the National Institute of Allergy and Infectious Disease to MS Smeltzer. DG Meeker was supported by T32 training grant GM106999. Support was also provided by the by the core facilities supported by the Center for Microbial Pathogenesis and Host Inflammatory Responses (P20-GM103450) and the Translational Research Institute (UL1TR000039) through the NIH National Center for Research Resources and National Center for Advancing Translational Sciences. The authors have no other relevant affiliations or financial involvement with any organization or entity with a financial interest in or financial conflict with the subject matter or materials discussed in the manuscript apart from those disclosed.

No writing assistance was utilized in the production of this manuscript.

4 Libutti SK, Paciotti GF, Byrnes AA et al. Phase I and pharmacokinetic studies of CYT-6091, a novel PEGylated colloidal Gold-rhTNF nanomedicine. Clin. Cancer Res. 16, 6139-6149 (2010).

5 Shao J, Griffin RJ, Galanzha EI et al. Photothermal nanodrugs: potential of TNF-gold nanospheres for cancer theranostics. Sci. Rep. 3, 1293 (2013). 
6 Hirsch LR, Stafford R, Bankson J et al. Nanoshell-mediated near-infrared thermal therapy of tumors under magnetic resonance guidance. Proc. Natl Acad. Sci. USA 100, 13549-13554 (2003).

7 Bobo D, Robinson KJ, Islam J, Thurecht KJ, Corrie SR. Nanoparticle-based medicines: a review of FDA-approved materials and clinical trials to date. Pharm. Res. doi:10.1007/ s11095-016-1958-5 (2016) (Epub ahead of print).

8 Zhu X, Radovic-Moreno AF, Wu J, Langer R, Shi J. Nanomedicine in the management of microbial infection - overview and perspectives. Nano Today 9(4), 478-498 (2014).

9 Boucher HW, Talbot GH, Benjamin DK Jr et al. 10 x '20 Progress - development of new drugs active against Gramnegative bacilli: an update from the Infectious Diseases Society of America. Clin. Infect. Dis. 56(12), 1685-1694 (2013).

10 Archer NK, Mazaitis MJ, Costerton JW, Leid JG, Powers ME, Shirtliff ME. Staphylococcus aureus biofilms: properties, regulation, and roles in human disease. Virulence 2(5), 445-459 (2011).

11 Weiss EC, Spencer HJ, Daily SJ, Weiss BD, Smeltzer MS. Impact of sarA on antibiotic susceptibility of Staphylococcus aureus in a catheter-associated in vitro model of biofilm formation. Antimicrob. Agent Chemother. 53(6), 2475-2482 (2009).

12 Weiss EC, Zielinska A, Beenken KE, Spencer HJ, Daily SJ, Smeltzer MS. Impact of $s a r A$ on daptomycin susceptibility of Staphylococcus aureus biofilms in vivo. Antimicrob. Agents Chemother. 53(10), 4096-4102 (2009).
13 Brady RA, Leid JG, Calhoun JH, Costerton JW, Shirtliff ME. Osteomyelitis and the role of biofilms in chronic infection. FEMS Immunol. Med. Micribiol. 52(1), 13-22 (2008).

14 Römling U, Balsalobre C. Biofilm infections, their resilience to therapy and innovative treatment strategies. J. Intern. Med. 272(6), 541-561 (2012).

15 Wang LS, Gupta A, Rotello VM. Nanomaterials for the treatment of bacterial biofilms. ACS Infect. Dis. 2, 3-4 (2015).

16 Zharov VP, Mercer KE, Galitovskaya EN, Smeltzer MS. Photothermal nanotherapeutics and nanodiagnostics for selective killing of bacteria targeted with gold nanoparticles. Biophys. J. 90(2), 619-627 (2006).

17 Galanzha EI, Shashkov E, Sarimollaoglu M et al. In vivo magnetic enrichment, photoacoustic diagnosis, and photothermal purging of infected blood using multifunctional gold and magnetic nanoparticles. PLoS One 7(9), e45557 (2012).

18 Etheridge ML, Campbell SA, Erdman AG, Haynes CL, Wolf $\mathrm{SM}, \mathrm{McCullough}$ J. The big picture on nanomedicine: the state of investigational and approved nanomedicine products. Nanomedicine 9(1), 1-14 (2013).

19 Yavuz MS, Cheng Y, Chen J et al. Gold nanocages covered by smart polymers for controlled release with near-infrared light. Nat. Mater. 8(12), 935-939 (2009).

20 Meeker DG, Jenkins SV, Miller EK et al. Synergistic photothermal and antibiotic killing of biofilm-associated Staphylococcus aureus using targeted antibiotic-loaded gold nanoconstructs. ACS Infect. Dis. 2, 241-250 (2016). 